TRANSACTIONS OF THE

AMERICAN MATHEMATICAL SOCIETY

Volume 358, Number 7, Pages 2855-2874

S 0002-9947(06)04157-2

Article electronically published on February 14, 2006

\title{
THE FLAT MODEL STRUCTURE ON COMPLEXES OF SHEAVES
}

\author{
JAMES GILLESPIE
}

\begin{abstract}
Let $\mathbf{C h}(\mathcal{O})$ be the category of chain complexes of $\mathcal{O}$-modules on a topological space $T$ (where $\mathcal{O}$ is a sheaf of rings on $T$ ). We put a Quillen model structure on this category in which the cofibrant objects are built out of flat modules. More precisely, these are the dg-flat complexes. Dually, the fibrant objects will be called dg-cotorsion complexes. We show that this model structure is monoidal, solving the previous problem of not having any monoidal model structure on $\mathbf{C h}(\mathcal{O})$. As a corollary, we have a general framework for doing homological algebra in the category $\operatorname{Sh}(\mathcal{O})$ of $\mathcal{O}$-modules. I.e., we have a natural way to define the functors Ext and Tor in $\operatorname{Sh}(\mathcal{O})$.
\end{abstract}

\section{INTRODUCTION}

Let $(\mathcal{O}, T)$ be a ringed space. That is, $T$ is a topological space and $\mathcal{O}$ is a sheaf of (commutative) rings (with 1 ) on $T$. In algebraic geometry we study the category $\mathbf{S h}(\mathcal{O})$ of sheaves of $\mathcal{O}$-modules. This category is a popular example of a Grothendieck category. Consequently, it has a lot of properties similar to the category $\mathbf{R m o d}$ of $R$-modules, where $R$ is a commutative ring with identity. One main difference though is that $\mathbf{S h}(\mathcal{O})$ does not have enough projective objects. This causes some difficulties when doing homotopy theory on the chain complex category $\mathbf{C h}(\mathcal{O})$ (i.e., doing homological algebra in $\mathbf{S h}(\mathcal{O})$ ). Surely we can compute $\operatorname{Ext}_{\mathcal{O}}^{n}(-,-)$ in $\mathbf{S h}(\mathcal{O})$ since Grothendieck categories have enough injectives. However, since we also have a tensor product on $\mathbf{S h}(\mathcal{O})$, we would also like a $\operatorname{Tor}_{\mathcal{O}}^{n}(-,-)$ functor analogous to the situation in $\mathbf{R m o d}$. (In fact, $\mathbf{S h}(\mathcal{O})$ is the same as $\mathbf{R m o d}$ when we take $T$ to be the one point space and let $\mathcal{O}$ be the constant sheaf with $\mathcal{O}(U)=R$ for all $U \subseteq T$.) Since we do not have enough projectives we cannot define it in the same way we do for $\mathbf{R m o d}$. The idea then is to use flat modules since they are tensor exact. But a flat resolution of a sheaf $S$ need not be unique up to homotopy equivalence. Consequently, we cannot uniquely define Tor using such flat resolutions. Edgar Enochs and L. Oyonarte recently showed we can define Tor by showing the existence of flat resolutions for which all the kernels are cotorsion EO01. Considering such resolutions allows us to show that any sheaf $S$ has a unique resolution up to homotopy equivalence.

Received by the editors January 8, 2004.

2000 Mathematics Subject Classification. Primary 55U35, 18G15.

The author thanks Mark Hovey of Wesleyan University and Edgar Enochs of the University of Kentucky.

(C)2006 American Mathematical Society Reverts to public domain 28 years from publication 
We will deal with the problem in a more general way and from a slightly different perspective. We take the point of view that wherever there exists a notion of homotopy in some category $\mathcal{D}$, then there should be a Quillen model structure Qui67 on $\mathcal{D}$ describing it. Furthermore, if $\mathcal{D}$ is a closed symmetric monoidal category (i.e., if $\mathcal{D}$ has a tensor product), then ideally we would like a monoidal model structure on $\mathcal{D}$. Loosely speaking, this just means the model structure is compatible with the tensor product.

It was shown in Gil04 that there is a new monoidal model structure on $\mathbf{C h}(R)$ which we call the "flat model structure". The point of this paper is to generalize this result to $\mathbf{C h}(\mathcal{O})$. Again, this is a much more interesting and useful result since $\operatorname{Sh}(\mathcal{O})$ does not have enough projectives. In model category terms, this means there is no projective model structure on $\mathbf{C h}(\mathcal{O})$ analogous to the well-known projective model structure on $\mathbf{C h}(R)$. There is an "injective model structure" on complexes of sheaves, but it is not monoidal [Joy84.

One useful corollary to having this flat model structure is that it will implicitly deal with the "flat resolution problem" mentioned above. The model structure will provide the "right type" of resolution, simply by taking a cofibrant replacement of the module $S$. In short, the flat model structure on $\operatorname{Ch}(\mathcal{O})$ gives us the proper framework to define the derived functors Ext and Tor using flat resolutions. The method boils down to taking resolutions by flat $\mathcal{O}$-modules so that the kernels are cotorsion. Therefore, the method of Enochs and Oyonarte mentioned above is encompassed in Quillen's idea of a monoidal model category.

\section{Preliminaries}

This section is meant to serve as a reference for definitions, notation, and theorems that we will refer to in later sections of the paper. In particular, we will discuss cotorsion pairs, which Hovey showed in Hov02 are closely related to model structures on abelian categories. For the rest of the paper we assume that the reader has a basic understanding of model categories (see [DS95, Hov99, and Qui67), Grothendieck categories (see [Sten75]), and sheaves (see Har77]). For much more information on cotorsion pairs see [EJ01].

Let $\mathcal{D}$ be an abelian category. A cotorsion pair (also called a cotorsion theory) is a pair of classes of objects $(\mathcal{A}, \mathcal{B})$ of $\mathcal{D}$ such that $\mathcal{A}^{\perp}=\mathcal{B}$ and $\mathcal{A}={ }^{\perp} \mathcal{B}$. Here $\mathcal{A}^{\perp}$ is the class of objects $X \in \mathcal{D}$ such that $\operatorname{Ext}^{1}(A, X)=0$ for all $A \in \mathcal{A}$, and similarly ${ }^{\perp} \mathcal{B}$ is the class of objects $X \in \mathcal{D}$ such that $\operatorname{Ext}^{1}(X, B)=0$ for all $B \in \mathcal{B}$. Two simple examples of cotorsion pairs in $\operatorname{Rmod}$ are $(\mathcal{P}, \mathcal{A})$ and $(\mathcal{A}, \mathcal{I})$, where $\mathcal{P}$ is the class of projectives, $\mathcal{I}$ is the class of injectives and $\mathcal{A}$ is the class of all $R$-modules. Another example of a cotorsion theory in $\operatorname{Rmod}$ is $(\mathcal{F}, \mathcal{C})$, where $\mathcal{F}$ is the class of flat modules and $\mathcal{C}$ are the so-called cotorsion modules. For a reference on cotorsion modules see [Xu96] and EJ01.

The cotorsion pair is said to have enough projectives if for any $X \in \mathcal{D}$ there is a short exact sequence $0 \rightarrow B \rightarrow A \rightarrow X \rightarrow 0$ where $B \in \mathcal{B}$ and $A \in \mathcal{A}$. We say it has enough injectives if it satisfies the dual statement. It is worth making a note on this terminology. The phrase "enough projectives" and "enough injectives" is standard in reference to cotorsion pairs. Unfortunately we also use the phrase "enough projectves/injectives" in reference to a category. This should never be confusing since from the context we are always referring to either a category or a cotorsion pair. Note however that saying that the projective cotorsion pair, $(\mathcal{P}, \mathcal{A})$, has 
enough projectives is equivalent to saying that the category has enough projectives. So in fact the terminology applied to a cotorsion theory is just a generalization of the usual terminology. In addition however, for any class of objects $\mathcal{F}$ in an abelian category $\mathcal{D}$, the author will use the terminology enough $\mathcal{F}$-objects to mean for any object $X \in \mathcal{D}$ there exists an $F \in \mathcal{F}$ and an epimorphism $F \rightarrow X$. Thus if $(\mathcal{F}, \mathcal{C})$ is the "flat" cotorsion pair described above, saying we have enough $\mathcal{F}$-objects means we can find a surjection $F \rightarrow M$ where $F$ is flat. But we say $(\mathcal{F}, \mathcal{C})$ has enough projectives to mean there exists a short exact sequence

$$
0 \rightarrow C \rightarrow F \rightarrow M \rightarrow 0
$$

where $C \in \mathcal{C}$ and $F \in \mathcal{F}$.

The central cotorsion pair we study in this paper is $(\mathcal{F}, \mathcal{C})$, where $\mathcal{F}$ is the set of all flat $\mathcal{O}$-modules and $\mathcal{C}=\mathcal{F}^{\perp}$ is the class of cotorsion $\mathcal{O}$-modules. An $\mathcal{O}$-module $F$ is called flat if the functor $F \otimes_{\mathcal{O}}-$ is an exact functor. Recall that for two $\mathcal{O}$ modules $S_{1}$ and $S_{2}$, we define $S_{1} \otimes_{\mathcal{O}} S_{2}$ to be the sheafification of the presheaf which assigns each open set $U \subseteq T$ to $S_{1}(U) \otimes_{\mathcal{O}(U)} S_{2}(U)$. We always assume our rings are commutative with identity 1 , so that $S_{1}(U) \otimes_{\mathcal{O}(U)} S_{2}(U)$ is an $\mathcal{O}(U)$-module and therefore $S_{1} \otimes_{\mathcal{O}} S_{2}$ is a sheaf of $\mathcal{O}$-modules. Like most properties of sheaves, flatness is a "stalkwise property". That is, $F$ is flat iff $F_{p}$ is a flat $\mathcal{O}_{p}$-module for each $p \in T$. This follows from the well-known isomorphism

$$
\left(S_{1} \otimes_{\mathcal{O}} S_{2}\right)_{p} \cong\left(S_{1}\right)_{p} \otimes_{\mathcal{O}_{p}}\left(S_{2}\right)_{p},
$$

which can be found in Lit82, Proposition 1.16. In $\mathbf{S h}(\mathcal{O})$, there are enough flat objects even though there are not enough projective objects. This fact is not hard, and the proof can be found as Proposition 1.2 in Har66.

We say a cotorsion theory is complete if it has enough projectives and enough injectives. The three cotorsion pairs in $\mathbf{R} \mathbf{m o d}$ described above are all examples of complete pairs. Proving that the "flat" pair $(\mathcal{F}, \mathcal{C})$ is complete is nontrivial, and two different proofs were recently given by the three authors of [BBE01]. Similarly we have the "flat" cotorsion pair on $\operatorname{Sh}(\mathcal{O})$, the category of sheaves of $\mathcal{O}$-modules where $\mathcal{O}$ is a ringed space on $T$. This cotorsion pair is also complete as follows from [EO01.

The next theorem is a special case of Theorem 2.2 from Hov02 which relates cotorsion pairs to model structures on abelian categories. It is this method which we will use to find the flat model structure on $\mathbf{C h}(\mathcal{O})$.

Theorem 2.1 (Hovey). Let $\mathcal{D}$ be an abelian category with all small limits and colimits and let $\mathbf{C h}(\mathcal{D})$ be the category of chain complexes on $\mathcal{D}$. Let $\mathcal{E}$ be the class of all exact chain complexes and let $\mathcal{Q}$ and $\mathcal{R}$ be classes of chain complexes such that $(\mathcal{Q}, \mathcal{R} \cap \mathcal{E})$ and $(\mathcal{Q} \cap \mathcal{E}, \mathcal{R})$ are complete cotorsion theories. Then there exists a model structure on $\mathbf{C h}(\mathcal{D})$, where the weak equivalences are $H_{*}$-isomorphisms, the cofibrations are the monomorphisms whose cokernels are in $\mathcal{Q}$ and the fibrations are the epimorphisms whose kernels are in $\mathcal{R}$.

Furthermore, in case $\mathcal{D}$ is a closed symmetric monoidal category, Hovey gives conditions on the classes $\mathcal{Q}$ and $\mathcal{R}$ which guarantee that the model structure is monoidal. So to find the flat model structure we will need to come up with the classes $\mathcal{Q}$ and $\mathcal{R}$. This leads us to the following definition which first appeared in Gil04. 
Definition 2.2. Let $(\mathcal{A}, \mathcal{B})$ be a cotorsion pair on an abelian category $\mathcal{D}$. Let $X$ be a chain complex.

(1) $X$ is called an $\mathcal{A}$ complex if it is exact and $Z_{n} X \in \mathcal{A}$ for all $n$.

(2) $X$ is called a $\mathcal{B}$ complex if it is exact and $Z_{n} X \in \mathcal{B}$ for all $n$.

(3) $X$ is called a $d g-\mathcal{A}$ complex if $X_{n} \in \mathcal{A}$ for each $n$, and every chain map $f: X \rightarrow B$ with $B$ a $\mathcal{B}$-complex, is homotopic to zero.

(4) $X$ is called a $d g-\mathcal{B}$ complex if $X_{n} \in \mathcal{B}$ for each $n$, and every chain map $f: A \rightarrow X$ with $A$ an $\mathcal{A}$-complex, is homotopic to zero.

We denote the class of $\mathcal{A}$ complexes by $\widetilde{\mathcal{A}}$ and the class of dg- $\mathcal{A}$ complexes by $d g \widetilde{\mathcal{A}}$. Similarly, the $\mathcal{B}$ complexes are denoted by $\widetilde{\mathcal{B}}$ and the class of $\mathrm{dg}-\mathcal{B}$ complexes are denoted by $d g \widetilde{\mathcal{B}}$. These definitions are inspired from the dg-projective complexes and projective complexes in $\mathbf{C h}(R)$, induced by the projective cotorsion pair.

Now let $\mathcal{G}$ be a Grothendieck category and let $(\mathcal{A}, \mathcal{B})$ be a cotorsion pair. Corollary 3.8 of Gil04 tells us that if $\mathcal{G}$ has enough $\mathcal{A}$-objects, then we have the induced cotorsion pairs $(\widetilde{\mathcal{A}}, d g \widetilde{\mathcal{B}})$ and $(d g \widetilde{\mathcal{A}}, \widetilde{\mathcal{B}})$ of chain complexes.

A cotorsion pair $(\mathcal{A}, \mathcal{B})$ is called hereditary if one of the following hold:

(1) $\mathcal{A}$ is resolving. That is, $\mathcal{A}$ is closed under taking kernels of epis.

(2) $\mathcal{B}$ is coresolving. That is, $\mathcal{B}$ is closed under taking cokernels of monics.

(3) $\operatorname{Ext}^{i}(A, B)=0$ for any $A \in \mathcal{A}$ and $B \in \mathcal{B}$ and $i \geq 1$.

See Theorem 1.2.10 of GR99 for a proof that these are equivalent.

If a cotorsion pair $(\mathcal{A}, \mathcal{B})$ is hereditary and our category $\mathcal{D}$ has enough projectives and injectives, then $d g \widetilde{\mathcal{A}} \cap \mathcal{E}=\widetilde{\mathcal{A}}$ and $d g \widetilde{\mathcal{B}} \cap \mathcal{E}=\widetilde{\mathcal{B}}$, where $\mathcal{E}$ be the class of all exact chain complexes Gil04. In this case we say the induced cotorsion pairs are compatible, and we have cotorsion pairs $(d g \widetilde{\mathcal{A}}, d g \widetilde{\mathcal{B}} \cap \mathcal{E})$ and $(d g \widetilde{\mathcal{A}} \cap \mathcal{E}, d g \widetilde{\mathcal{B}})$. This makes the situation reminiscent of the hypothesis of Theorem 2.1 by making $\mathcal{Q}=d g \widetilde{\mathcal{A}}$ and $\mathcal{R}=d g \widetilde{\mathcal{B}}$. But for a general Grothendieck category $\mathcal{G}$, we may not have enough projectives. Therefore, to get the analogous result for hereditary cotorsion pairs in $\mathcal{G}$ we will need a slight modification. This will be provided in Corollary 3.7.

The typical cotorsion pairs one comes across in practice are hereditary. For example, all of the previous examples of cotorsion pairs are hereditary. For the "flat" cotorsion pair $(\mathcal{F}, \mathcal{C})$ in $\operatorname{Sh}(\mathcal{O})$, a typical tensor product argument (like the one found in Proposition 3.4 of [Lan97]) will show that this is a hereditary cotorsion pair.

\section{A Simplification FOR GRothendieck CATEGories}

For the rest of the paper we let $(\mathcal{F}, \mathcal{C})$ denote the "flat" cotorsion pair in the category $\operatorname{Sh}(\mathcal{O})$. As we have already noted, it follows from Gil04 that we have two induced cotorsion pairs in $\mathbf{C h}(\mathcal{O})$, which we denote $(d g \widetilde{\mathcal{F}}, \widetilde{\mathcal{C}})$ and $(\widetilde{\mathcal{F}}, d g \widetilde{\mathcal{C}})$. We will call the complexes in the class $\widetilde{\mathcal{F}}$, flat, and the complexes in the class $d g \widetilde{\mathcal{F}}, d g$ flat. Similarly, we will use the terminology cotorsion and $d g$-cotorsion. To obtain the "flat" model structure we will use Hovey's Theorem 2.2 from [Hov02, which we have reprinted in section 2 in a more convenient form as Theorem 2.1. Thus we wish to show first that $(d g \widetilde{\mathcal{F}}, \widetilde{\mathcal{C}})$ and $(\widetilde{\mathcal{F}}, d g \widetilde{\mathcal{C}})$ are complete and second that $(d g \widetilde{\mathcal{F}}, \widetilde{\mathcal{C}})$ and $(\widetilde{\mathcal{F}}, d g \widetilde{\mathcal{C}})$ are compatible. By compatible we mean that $d g \widetilde{\mathcal{F}} \cap \mathcal{E}=\widetilde{\mathcal{F}}$ and $d g \widetilde{\mathcal{C}} \cap \mathcal{E}=\widetilde{\mathcal{C}}$, where $\mathcal{E}$ is the class of exact complexes. 
In this section we solve the problem of showing that the induced cotorsion pair $(d g \widetilde{\mathcal{F}}, \widetilde{\mathcal{C}})$ is complete. We also show that $(d g \widetilde{\mathcal{F}}, \widetilde{\mathcal{C}})$ and $(\widetilde{\mathcal{F}}, d g \widetilde{\mathcal{C}})$ are compatible. Since the solutions are very general and could possibly be used for other situations, we prove it for an arbitrary Grothendieck category with reasonable hypotheses on the cotorsion pair.

In particular, we let $(\mathcal{A}, \mathcal{B})$ be a hereditary cotorsion pair and let $\mathcal{G}$ be a Grothendieck category with enough $\mathcal{A}$-objects. Again, this means that for any object $X \in \mathcal{G}$, we have an epimorphism $A \rightarrow X$ where $A \in \mathcal{A}$. We will also assume that $(\mathcal{A}, \mathcal{B})$ is cogenerated by a set $\mathcal{S}$. A cotorsion pair $(\mathcal{A}, \mathcal{B})$ is cogenerated by a set if there is a set $\mathcal{S} \subseteq \mathcal{A}$ (so not just a class) such that $\mathcal{S}^{\perp}=\mathcal{C}$. This idea is fundamental to the study of cotorsion pairs. It will come up again in section 4 These hypotheses are all satisfied by the "flat" cotorsion pair in $\operatorname{Sh}(\mathcal{O})$. For example, to see that the cotorsion pair is cogenerated by a set, see the proof of Theorem 3.1 in [EO01].

One thing that makes a Grothendieck category feel more "concrete" is the fact that every object is $\kappa$-presented, where $\kappa$ is some infinite cardinal. Since we will use this idea heavily we start with some definitions.

Definition 3.1. Let $\kappa$ be a cardinal number. A nonempty category $\mathcal{K}$ is called $\kappa$ filtered if every small subcategory $\mathcal{S} \subseteq \mathcal{K}$ with $|\operatorname{mor}(\mathcal{S})| \leq \kappa$ is the base of a cocone. That is, there is an object $X$ and arrows $f_{S}: S \rightarrow X$ (one for each $S \in \mathcal{S}$ ) such that $f_{T} \circ j=f_{S}$ whenever $j: S \rightarrow T$ is in $\operatorname{mor}(\mathcal{S})$. We call a functor $F: \mathcal{K} \rightarrow \mathcal{D}$ a $\kappa$-filtered functor if $\mathcal{K}$ is a $\kappa$-filtered category. The colimit of a $\kappa$-filtered functor $F: \mathcal{K} \rightarrow \mathcal{D}$ is called a $\kappa$-filtered colimit.

One specialization of the idea of a $\kappa$-filtered category is the notion of a $\kappa$-filtered ordinal. (For example, see Hov99, p. 28.) Of course this is an ordinal $\alpha$ in which for any $S \subset \alpha$ with $|S| \leq \kappa$, we have $\sup S<\alpha$ (or to say the same thing, $\bigcup S \in \alpha$ ). It is a fact that given any infinite cardinal $\kappa$, the smallest $\kappa$-filtered limit ordinal is $\kappa^{+}$, the cardinal successor of $\kappa$. Also, any successor cardinal $\kappa^{\prime}>\kappa$ is $\kappa$-filtered.

Given a functor $F: \mathcal{K} \rightarrow \mathcal{D}$ and an object $X \in \mathcal{D}$, we will denote the composition of $\operatorname{Hom}_{\mathcal{D}}(X,-)$ with $F$ by $\operatorname{Hom}_{\mathcal{D}}(X, F)$. Recall that there is a natural map

$$
\operatorname{colim}_{\operatorname{Hom}_{\mathcal{D}}}(X, F) \rightarrow \operatorname{Hom}_{\mathcal{D}}(X, \operatorname{colim} F)
$$

(assuming all of these colimits exist) induced by the universal property of a colimit.

Definition 3.2. Let $\kappa$ be a cardinal and let $X$ be an object of a category $\mathcal{D}$. We say $X$ is $\kappa$-presented (or $\kappa$-small) if for any $\kappa$-filtered functor $F: \mathcal{K} \rightarrow \mathcal{D}$ (whose colimit exists in $\mathcal{D})$, the natural map $\operatorname{colim} \operatorname{Hom}_{\mathcal{D}}(X, F) \rightarrow \operatorname{Hom}_{\mathcal{D}}(X, \operatorname{colim} F)$ is an isomorphism.

In particular, suppose $\alpha$ is a $\kappa$-filtered ordinal and

$$
X_{0} \subseteq X_{1} \subseteq \cdots \subseteq X_{\gamma} \subseteq \cdots
$$

is an increasing sequence of objects indexed by $\alpha$. If $X$ is $\kappa$-presented, then any map $X \rightarrow \bigcup X_{\gamma}$ factors through some $X_{\gamma}$. Also, note that if $\kappa^{\prime} \geq \kappa$, then a $\kappa$-presented object $X \in \mathcal{D}$ is also $\kappa^{\prime}$-presented.

Now we recall a lemma concerning injective objects which the author learned from Gro57. One can also see Proposition 2.9 in Chapter V of Sten75 for a proof. 
Lemma 3.3 (Grothendieck). If $\mathcal{G}$ is a Grothendieck category, then an object $I \in \mathcal{G}$ is injective iff for each subobject $V \subset U$, where $U$ is a generator, and each morphism $f: V \rightarrow I, f$ extends to a morphism $U \rightarrow I$.

In a Grothendieck category, $\mathcal{G}$, every object is $\kappa$-presented for some $\kappa$ Hov01. Also the class of subobjects of an object is actually a set. (See [Sten75, p. 94). Now if we let $U$ be a generator for $\mathcal{G}$, then each subobject $V \subseteq U$ is $\kappa_{V}$-presented for some cardinal $\kappa_{V}$. Let $\kappa=\sup \left\{\kappa_{V}: V \subseteq U\right\}$. Then clearly all $U$ and all of its subobjects are $\kappa$-presented. Now if we let $\mathcal{K}$ be a $\kappa$-filtered category, and consider a functor $F: \mathcal{K} \rightarrow \mathcal{G}$ where $F(d)$ is injective for all $i \in \mathcal{K}$, then for any subobject $V \subset U$, and arrow $i \rightarrow j$ in $\mathcal{K}$, we get a commutative diagram

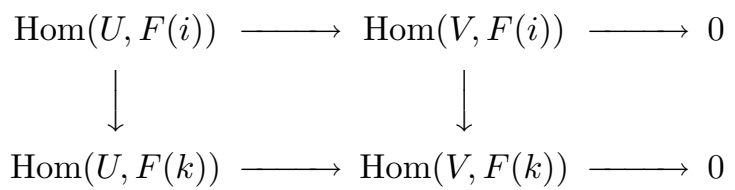

with exact rows. Thus

$$
\operatorname{colim} \operatorname{Hom}(U, F) \rightarrow \operatorname{colim} \operatorname{Hom}(V, F) \rightarrow 0
$$

is exact. Also, since $\mathcal{K}$ is $\kappa$-filtered, the direct limits commute to give us that

$$
\operatorname{Hom}(U, \operatorname{colim} F) \rightarrow \operatorname{Hom}(V, \operatorname{colim} F) \rightarrow 0
$$

is exact. So Grothendieck's Lemma 3.3 implies the following lemma.

Lemma 3.4. Let $\mathcal{G}$ be a Grothendieck category. Then there exists a cardinal $\kappa$ such that for every $\kappa$-filtered functor $F: \mathcal{K} \rightarrow \mathcal{G}$ with $F(j)$ injective for all $j \in \mathcal{K}$, we have colim $F$ injective.

Lemma 3.5. Let $\mathcal{G}$ be a Grothendieck category and let $(\mathcal{A}, \mathcal{B})$ be a cotorsion pair cogenerated by a set $\mathcal{S}$. Then there exists a cardinal $\kappa^{\prime}$ such that for every $\kappa^{\prime}$-filtered functor $F: \mathcal{K} \rightarrow \mathcal{G}$ with $F(j) \in \mathcal{B}$ for all $j \in \mathcal{K}$, we have $\operatorname{colim} F \in \mathcal{B}$.

Proof. Since $(\mathcal{A}, \mathcal{B})$ is cogenerated by a set $\mathcal{S}$, there actually exists a single object $A$ which cogenerates $(\mathcal{A}, \mathcal{B})$. I.e. $\operatorname{Ext}^{1}(A, B)=0$ iff $B \in \mathcal{B}$. Let $\kappa$ be as in the last lemma and let $\kappa^{\prime} \geq \kappa$ be a cardinal for which $A$ is $\kappa^{\prime}$-presented.

Now let $\mathcal{K}$ be a $\kappa^{\prime}$-filtered category and let $F: \mathcal{K} \rightarrow \mathcal{G}$ be a functor such that $F(j) \in \mathcal{B}$ for all $j \in \mathcal{K}$. It follows from Corollary 6.6 of $[\mathrm{Hov} 02$ that in a Grothendieck category we may take injective coresolutions functorially. Therefore, we may take a functorial injective coresolution of the diagram $F(\mathcal{K})$ as shown below:

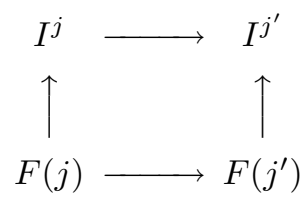

Applying the functor $\operatorname{Hom}(A,-)$ to the diagram of injective complexes $\left\{I^{j}\right\}_{j \in \mathcal{K}}$ gives us (in the obvious way) a diagram of complexes $\left\{\operatorname{Hom}\left(A, I^{j}\right)\right\}_{j \in \mathcal{K}}$ By hypothesis $\operatorname{Ext}^{1}(A, F(j))=H^{1} \operatorname{Hom}\left(A, I^{j}\right)=0$ for all $j \in \mathcal{K}$. So it follows that $H^{1}\left[\operatorname{colim}_{j \in \mathcal{K}} \operatorname{Hom}\left(A, I^{j}\right)\right]=0$. But since $\mathcal{K}$ is $\kappa$-filtered, the last lemma tells us that colim $F \rightarrow \operatorname{colim}_{j \in \mathcal{K}} I^{j}$ is an injective coresolution. Also, since $\mathcal{K}$ is $\kappa^{\prime}$-filtered,

$$
\operatorname{Hom}\left(A, \operatorname{colim}_{j \in \mathcal{K}} I^{j}\right) \cong \operatorname{colim}_{j \in \mathcal{K}} \operatorname{Hom}\left(A, I^{j}\right) .
$$


Therefore,

$$
\operatorname{Ext}^{1}(A, \operatorname{colim} F)=H^{1}\left[\operatorname{Hom}\left(A, \operatorname{colim}_{j \in \mathcal{K}} I^{j}\right)\right]=0
$$

For a moment, let $\mathcal{D}$ be any cocomplete abelian category (in particular, not necessarily Grothendieck). Though it is an abuse of notation, we will also let $\mathcal{D}$ denote the class of objects of $\mathcal{D}$. Then for a complete cotorsion theory $(\mathcal{A}, \mathcal{B})$, clearly $(\mathcal{A}, \mathcal{B})=(\mathcal{A} \cap \mathcal{D}, \mathcal{B})=(\mathcal{A}, \mathcal{B} \cap \mathcal{D})$. So Hovey's Theorem 2.2 from Hov02 gives us a (trivial) model structure on the category $\mathcal{D}$ with the cofibrations being the injective maps $i$ with $\operatorname{cok} i \in \mathcal{A}$ and the fibrations being the surjective maps $p$ with $\operatorname{ker} p \in \mathcal{B}$. In particular, any map $f: X \rightarrow Y$ factors as $f=p i$, where $i$ is a cofibration and $p$ is a fibration. We will use this to prove the next proposition. The author learned the idea of the proof from Edgar Enochs.

Proposition 3.6. Let $(\mathcal{A}, \mathcal{B})$ be a cotorsion pair in a Grothendieck category $\mathcal{G}$ with enough $\mathcal{A}$-objects. Furthermore assume that $(\mathcal{A}, \mathcal{B})$ is cogenerated by a set. Then the cotorsion pair of complexes $(d g \widetilde{\mathcal{A}}, \widetilde{\mathcal{B}})$ has enough injectives.

Proof. The assumption that $\mathcal{G}$ has enough $\mathcal{A}$-objects is only used to guarantee that $(d g \widetilde{\mathcal{A}}, \widetilde{\mathcal{B}})$ is indeed a cotorsion pair. (See Corollary 3.8 of Gil04.)

Let $X$ be any complex. We want to embed $X$ as $0 \rightarrow X \rightarrow B \rightarrow A \rightarrow 0$ where $B \in \widetilde{\mathcal{B}}$ and $A \in d g \widetilde{\mathcal{A}}$. First let $\lambda$ be any ordinal and we will describe a general embedding method. Later we will specify an ordinal which will prove the proposition.

Let $\left(n_{k}\right)_{k \in \mathbb{N}}$ be any sequence which maps $\mathbb{N}$ bijectively onto $\mathbb{Z}$. Set $X^{0}=X$. Now factor $X_{n_{1}}^{0} \stackrel{d_{n_{1}}}{\longrightarrow} Z_{n_{1}-1}\left(X^{0}\right)$ as $p_{n_{1}} i_{n_{1}}$, where $p_{n_{1}}: Y_{n_{1}}^{0} \rightarrow Z_{n_{1}-1}\left(X^{0}\right)$ is a surjection with $\operatorname{ker} p_{n_{1}} \in \mathcal{B}$ and $i_{n_{1}}: X_{n_{1}}^{0} \rightarrow Y_{n_{1}}^{0}$ is an injection with cok $i_{n_{1}} \in \mathcal{A}$. Then set

$$
X^{1}=\cdots \rightarrow X_{n_{1}+1}^{0} \stackrel{i_{n_{1}} d}{\longrightarrow} Y_{n_{1}}^{0} \stackrel{p_{n_{1}}}{\longrightarrow} X_{n_{1}-1}^{0} \rightarrow \cdots .
$$

Then $X^{1}$ satisfies

(1) $Z_{n_{1}}\left(X^{1}\right)=\operatorname{ker} p_{n_{1}}$.

(2) $H_{n_{1}-1}\left(X^{1}\right)=0$.

(3) $X^{0} \subseteq X^{1}$ and $X^{1} / X^{0}=\cdots \rightarrow 0 \rightarrow Y_{n_{1}}^{0} / X_{n_{1}}^{0} \rightarrow 0 \rightarrow \cdots$ is a dg- $\mathcal{A}$ complex.

Using this method, we continue by transfinite induction to build an increasing sequence of chain complexes. Suppose $\alpha$ is an ordinal and suppose that we have already constructed

$$
X=X^{0} \subseteq X^{1} \subseteq X^{2} \subseteq \cdots \subseteq X^{\beta} \subseteq \cdots
$$

for all $\beta<\alpha$. If $\alpha$ is a limit ordinal, then we set $\alpha=\bigcup_{\beta<\alpha} X^{\beta}$. If $\alpha$ is a successor ordinal, we note that $\alpha$ has a unique representation as $\alpha=\gamma+k$, where $\gamma$ is a limit ordinal (or $\gamma=0$ when $\alpha$ is finite) and $k$ is finite. Then we construct $X^{\alpha}$ from $X^{\alpha-1}$ as follows: We factor $X_{n_{k}}^{\alpha-1} \stackrel{d_{n_{k}}}{\longrightarrow} Z_{n_{k}-1}\left(X^{\alpha-1}\right)$ as $p_{n_{k}} i_{n_{k}}$, where $p_{n_{k}}: Y_{n_{k}}^{\alpha-1} \rightarrow Z_{n_{k}-1}\left(X^{\alpha-1}\right)$ is a surjection with $\operatorname{ker} p_{n_{k}} \in \mathcal{B}$ and $i_{n_{k}}: X_{n_{k}}^{\alpha-1} \rightarrow$ $Y_{n_{k}}^{\alpha-1}$ is an injection with $\operatorname{cok} i_{n_{k}} \in \mathcal{A}$. Then set

$$
X^{\alpha}=\cdots \rightarrow X_{n_{k}+1}^{\alpha-1} \stackrel{i_{n_{k}} d}{\longrightarrow} Y_{n_{k}}^{\alpha-1} \stackrel{p_{n_{k}}}{\longrightarrow} X_{n_{k}-1}^{\alpha-1} \rightarrow \cdots .
$$

Therefore, $X^{\alpha}$ satisfies

(1) $Z_{n_{k}}\left(X^{\alpha}\right)=\operatorname{ker} p_{n_{k}}$. 
(2) $H_{n_{k}-1}\left(X^{\alpha}\right)=0$.

(3) $X^{\alpha-1} \subseteq X^{\alpha}$ and $X^{\alpha} / X^{\alpha-1}=\cdots \rightarrow 0 \rightarrow Y_{n_{k}}^{\alpha-1} / X_{n_{k}}^{\alpha-1} \rightarrow 0 \rightarrow \cdots$ is a $\operatorname{dg}-\mathcal{A}$ complex.

This completes the induction, and so for any ordinal $\lambda$ we get a continuous chain

$$
X=X^{0} \subseteq X^{1} \subseteq X^{2} \subseteq \cdots \subseteq X^{\alpha} \subseteq \cdots
$$

for all $\alpha<\lambda$. Finally we set $X^{\lambda}=\bigcup_{\alpha<\lambda} X^{\alpha}$.

Now let $\kappa^{\prime}$ be the cardinal guaranteed in the statement of Lemma 3.5. Let $\lambda$ be a limit ordinal such that the well-ordered set of all limit ordinals $\gamma<\lambda$ is isomorphic to a $\kappa^{\prime}$-filtered ordinal. (Given any ordinal $\alpha$, we can actually find a limit ordinal $\beta$ for which $\alpha$ is isomorphic to the well-ordered set of all limit ordinals less than $\beta$.) Then we claim that the short exact sequence $0 \rightarrow X \rightarrow X^{\lambda} \rightarrow X^{\lambda} / X \rightarrow 0$ is the desired short exact sequence.

First we show $X^{\lambda}$ is a $\mathcal{B}$ complex. Since $X=X^{0} \subseteq X^{1} \subseteq \cdots$ is increasing, for any natural number $k \in \mathbb{N}$ we may write

$$
X^{\lambda}=\bigcup_{\alpha<\lambda} X^{\alpha}=\bigcup_{\gamma<\lambda} X^{\gamma+k}
$$

where the last union is taken over all limit ordinals $\gamma<\lambda$. Since each $X^{\gamma+k}$ is exact in degree $n_{k}-1$, we see that the union $\bigcup X^{\gamma+k}$ is also exact at the $n_{k}-1$ spot. Since $k$ is arbitrary, $X^{\lambda}$ is exact. Next we want to see that each $Z_{k}\left(X^{\lambda}\right) \in \mathcal{B}$. But $Z_{k}\left(X^{\lambda}\right)=Z_{k}\left(\bigcup X^{\gamma+k}\right)=\bigcup Z_{k}\left(X^{\gamma+k}\right)$, where again the union is taken over all limit ordinals $\gamma<\lambda$. Now by construction each $Z_{k}\left(X^{\gamma+k}\right) \in \mathcal{B}$, and so by our choice of $\lambda, \bigcup Z_{k}\left(X^{\gamma+k}\right) \in \mathcal{B}$ by Lemma 3.5

Lastly, we need to argue that $X^{\lambda} / X$ is a dg- $\mathcal{A}$ complex. But $X^{\lambda} / X$ is a continuous union of the sequence

$$
X^{1} / X^{0} \subseteq X^{2} / X^{0} \subseteq \cdots \subseteq X^{\alpha+1} / X^{\alpha} \subseteq \cdots .
$$

Since $X^{\alpha+1} / X^{\alpha}$ is a dg- $\mathcal{A}$ complex for all $\alpha$, this shows that $X^{\lambda} / X^{0}$ is a transfinite extension of $d g \widetilde{\mathcal{A}}$. Since $d g \widetilde{\mathcal{A}}$ is a left cotorsion class, it is closed under transfinite extensions (for example, see Lemma 6.2 of [Hov02]), and so we are done.

Corollary 3.7. Let $\mathcal{G}$ be a Grothendieck category with enough $\mathcal{A}$ objects, where $(\mathcal{A}, \mathcal{B})$ is a hereditary cotorsion pair which is cogenerated by a set. Then the induced cotorsion pairs of chain complexes are compatible. That is, $d g \widetilde{\mathcal{A}} \cap \mathcal{E}=\widetilde{\mathcal{A}}$ and $d g \widetilde{\mathcal{B}} \cap \mathcal{E}=\widetilde{\mathcal{B}}$, where $\mathcal{E}$ is the class of all exact complexes. Furthermore, $($ dg $\widetilde{\mathcal{A}}, \widetilde{\mathcal{B}})$ is complete.

Proof. Since a Grothendieck category always has enough injectives, Theorem 3.12 from Gil04 tells us that $d g \widetilde{\mathcal{A}} \cap \mathcal{E}=\widetilde{\mathcal{A}}$. By Proposition 3.6 we know $(d g \widetilde{\mathcal{A}}, \widetilde{\mathcal{B}})$ has enough injectives. So Lemma 3.14 of [Gil04] tells us that $d g \widetilde{\mathcal{B}} \cap \mathcal{E}=\widetilde{\mathcal{B}}$.

To show $(d g \widetilde{\mathcal{A}}, \widetilde{\mathcal{B}})$ is complete it is only left to show that $(d g \widetilde{\mathcal{A}}, \widetilde{\mathcal{B}})$ has enough projectives. For that, we use the fact that $\mathcal{G}$ has enough $\mathcal{A}$-objects. Indeed, by Lemma 3.5 of [Gil04], $\mathbf{C h}(\mathcal{G})$ has enough $\widetilde{\mathcal{A}}$-objects. Therefore given any $X \in \mathcal{G}$ we have a short exact sequence

$$
0 \rightarrow K \rightarrow A \rightarrow X \rightarrow 0,
$$

where $A \in \widetilde{\mathcal{A}}$. Using Proposition 3.6. we have a short exact sequence

$$
0 \rightarrow K \rightarrow B \rightarrow A^{\prime} \rightarrow 0,
$$


where $A^{\prime} \in d g \widetilde{\mathcal{A}}$ and $B \in \widetilde{\mathcal{B}}$. Now form the commutative diagram below, where $P$ is a pushout:

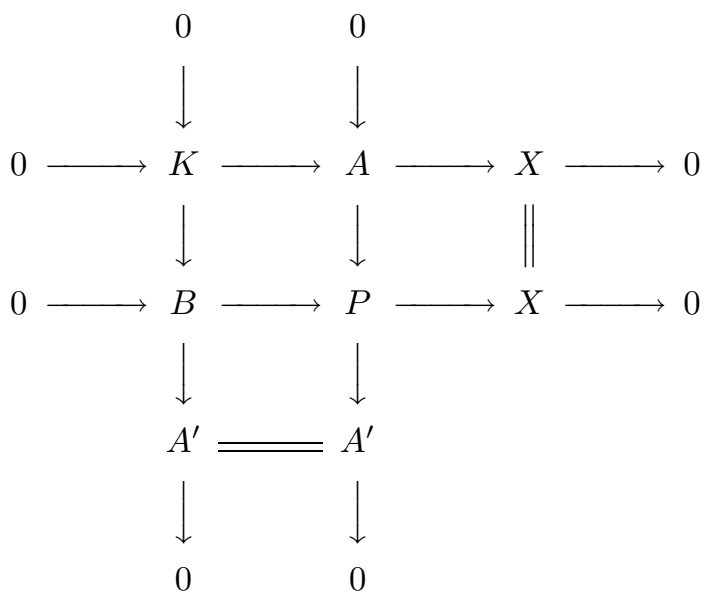

Since $A, A^{\prime} \in d g \widetilde{\mathcal{A}}$ and $d g \widetilde{\mathcal{A}}$ is closed under extensions, we see that $P \in d g \widetilde{\mathcal{A}}$. Therefore the short exact sequence

$$
0 \rightarrow B \rightarrow P \rightarrow X \rightarrow 0
$$

shows that $(d g \widetilde{\mathcal{A}}, \widetilde{\mathcal{B}})$ is has enough projectives.

Corollary 3.8. Let $\mathcal{G}$ be a Grothendieck category with enough $\mathcal{A}$ objects, where $(\mathcal{A}, \mathcal{B})$ is a hereditary cotorsion pair which is cogenerated by a set. If $(\widetilde{\mathcal{A}}, d g \widetilde{\mathcal{B}})$ has enough injectives, then we have an induced model category structure on $\mathbf{C h}(\mathcal{G})$, where the weak equivalences are the homology isomorphisms. The cofibrations are monomorphisms whose cokernels are in $d g \widetilde{\mathcal{A}}$, and the fibrations are the epimorphisms whose kernels are in $d g \widetilde{\mathcal{B}}$.

Proof. From Corollary 3.7 and Hovey's correspondence Theorem 2.1, the problem reduces to showing that $(\widetilde{\mathcal{A}}, d g \widetilde{\mathcal{B}})$ has enough projectives. This follows by using the same pushout argument as in the proof of Corollary 3.7 .

\section{The Flat Model StruCture}

In this section we show that the "flat" cotorsion pair $(\mathcal{F}, \mathcal{C})$ in $\mathbf{S h}(\mathcal{O})$ does in fact induce a model structure on $\operatorname{Ch}(\mathcal{O})$. Now $\mathbf{S h}(\mathcal{O})$ is a Grothendieck category, and as we mentioned in section $2(\mathcal{F}, \mathcal{C})$ is a hereditary cotorsion pair and $\operatorname{Sh}(\mathcal{O})$ has enough $\mathcal{F}$-objects. Furthermore, we can see from [EO01] (see its proof of Theorem 3.1) that $(\mathcal{F}, \mathcal{C})$ is cogenerated by a set. Therefore, due to Corollary 3.8, we just need to show that the cotorsion pair $(\widetilde{\mathcal{F}}, d g \widetilde{\mathcal{C}})$ has enough injectives. We tackle the problem in the standard way. We will show that $(\widetilde{\mathcal{F}}, d g \widetilde{\mathcal{C}})$ is cogenerated by a set and use the following proposition.

Proposition 4.1. Let $\mathcal{G}$ be any Grothendieck category and let $(\mathcal{A}, \mathcal{B})$ be a cotorsion pair cogenerated by a set. Then $(\mathcal{A}, \mathcal{B})$ has enough injectives.

Proposition 4.1 first appeared in Eklof and Trlifaj's paper [ET01, but only for the case $\mathcal{G}=\mathbf{R m o d}$. It was quickly used by Edgar Enochs to settle his "flat cover conjecture". Eklof and Trlifaj's proof readily generalizes to abelian categories with enough projectives and injectives. Recently however, some authors have noted that 
the result is true in any Grothendieck category. For example, see [EEGO]. Mark Hovey has also shown the author a different proof while pointing out that the lemma is a disguised form of Quillen's "small object argument" Qui67.

We make one last simplification of the problem before we actually dig into the category of sheaves of $\mathcal{O}$-modules.

Lemma 4.2. Let $\mathcal{G}$ be a Grothendieck category and let $(\mathcal{A}, \mathcal{B})$ be a cotorsion theory for which there exists a set $\mathcal{L} \subseteq \mathcal{A}$ such that for every $0 \neq A \in \mathcal{A}$, there exists a $0 \neq P \subseteq A$ with $P \in \mathcal{L}$, and $A / P \in \mathcal{A}$. Then the set $\mathcal{L}$ cogenerates $(\mathcal{A}, \mathcal{B})$.

Proof. By Lemma 4.5 of [Gil04, it is enough to show that everything in $\mathcal{A}$ is a transfinite extension of objects in $\mathcal{L}$. So given $0 \neq A \in \mathcal{A}$, pick $0 \neq P_{0} \subseteq A$ such that $P_{0} \in \mathcal{L}$ and $A / P_{0} \in \mathcal{A}$. If $A / P_{0} \neq 0$, pick $0 \neq P_{1} / P_{0} \subseteq A / P_{0}$ such that $P_{1} / P_{0} \in \mathcal{L}$ and $A / P_{1} \in \mathcal{A}$. Now we continue and use transfinite induction. For a limit ordinal $\gamma$, we define $P_{\gamma}=\bigcup_{\alpha<\gamma} P_{\alpha}$. For a successor ordinal $\alpha$, we find $P_{\alpha} / P_{\alpha-1} \subseteq A / P_{\alpha-1}$ just like above. Eventually this process must terminate, since we are in a Grothendieck category and every object has a set's worth of subobjects [Sten75]. In short, we end up with $A=\bigcup_{\alpha<\lambda} P_{\alpha}$ for some ordinal $\lambda$ and with $P_{0}, P_{\alpha+1} / P_{\alpha} \in \mathcal{L}$. So $F$ is a transfinite extension of objects in $\mathcal{L}$.

For reference we now record the definition of flat which we discussed in section 2 ,

Definition 4.3. Let $(\mathcal{O}, T)$ be a ringed space. A sheaf of $\mathcal{O}$-modules, $F$, is called flat if each stalk is flat. That is, for each $p \in T, \operatorname{colim}_{p \in U} F(U)=F_{p}$ is a flat $\mathcal{O}_{p}$-module.

Suppose $X$ is a chain complex of $\mathcal{O}$-modules (or presheaves of $\mathcal{O}$-modules). Then for each $p \in T$, we get a chain complex $X_{p}$ of $\mathcal{O}_{p}$-modules. If $X$ is a complex of sheaves, then unless explicitly stated otherwise, we call $X$ exact if for every $p \in T, X_{p}$ is exact. Also, if $X$ is a chain complex of presheaves, then unless stated otherwise, we say $X$ is exact if it is exact for each open set $U \subseteq T$. In particular, it is important to realize that a sheaf exact complex $X$ may not be presheaf exact.

Definition 4.4. We call a chain complex of $\mathcal{O}$-modules, $F$, flat if it is exact and each cycle $Z_{n} F$ is a flat $\mathcal{O}$-module. This definition agrees with the definition given in Definition 2.2 using the flat cotorsion theory in $\mathbf{S h}(\mathcal{O})$.

Recall that for a ring $R$ and an $R$-module $M$, we call a submodule $N \subseteq M$ pure if for any $R$-module $L$, the sequence $0 \rightarrow L \otimes_{R} N \rightarrow L \otimes_{R} M$ is exact.

Definition 4.5. Let $P \subseteq S$, where $S$ is an $\mathcal{O}$-module but $P$ is merely a (sub)presheaf of $\mathcal{O}$-modules. We say $P$ is presheaf pure in $S$ if for every open set $U \subseteq T$, $P(U)$ is a pure $\mathcal{O}(U)$-submodule of $S(U)$. We say $P$ is stalkwise pure in $S$ if for each $p \in T, P_{p}$ is a pure $\mathcal{O}_{p}$-submodule of $S_{p}$. If $P$ is in fact a sheaf, we will use the same terminology.

Not surprisingly, if $P \subseteq S$ is presheaf pure, then it is also stalkwise pure, as the next lemma shows.

Lemma 4.6. Let $P \subseteq S$, where $S$ is an $\mathcal{O}$-module. If $P$ is presheaf pure in $S$, then it is stalkwise pure in $S$.

Proof. Suppose $P \subseteq S$ is presheaf pure. Let $p \in T$. We know $P_{p} \rightarrow S_{p}$ is an injection, and we would like to see that it is a pure injection of $\mathcal{O}_{p}$-modules. So let 
$M$ be any $\mathcal{O}_{p}$-module and form the skyscraper sheaf $S_{p, M}$. This sheaf is defined by $U \mapsto M$ if $p \in U$ and $U \mapsto 0$ otherwise. It is in fact an $\mathcal{O}_{p}$-module by viewing $M$ as an $\mathcal{O}(U)$-module via the ring homomorphism $\mathcal{O}(U) \rightarrow \mathcal{O}_{p}$. Furthermore, it has the property that $\left(S_{p, M}\right)_{p}=M$. Now applying the presheaf tensor product $S_{p, M} \otimes-$ we get a monomorphism $S_{p, M} \otimes P \rightarrow S_{p, M} \otimes S$. This implies $\left(S_{p, M} \otimes P\right)_{p} \rightarrow\left(S_{p, M} \otimes S\right)_{p}$ is injective. This in turn implies $M \otimes_{\mathcal{O}_{p}} P_{p} \rightarrow M \otimes_{\mathcal{O}_{p}} S_{p}$ is injective since, in general, the stalk of the tensor product is the tensor product of the stalks.

Lemma 4.7. If $P$ is either presheaf pure or stalkwise pure in an $\mathcal{O}$-module $S$, then the sheafification, $P^{+}$, is stalkwise pure in $S$.

Proof. By Lemma 4.6 it is enough to assume $P$ is stalkwise pure. But if $P_{p} \subseteq S_{p}$ is pure, then $P_{p}^{+} \subseteq S_{p}$ is pure since $P_{p} \cong P_{p}^{+}$.

Define the cardinality of a presheaf/sheaf, $S$, to be $|S|=\left|\coprod_{U \subseteq T} S(U)\right|$. We now state a proposition of Enochs and Oyonarte which will be important in showing that the flat cotorsion pair $(\mathcal{F}, \mathcal{C})$ is cogenerated by a set. The following is Proposition 2.4 of [EO01].

Proposition 4.8. Let $(\mathcal{O}, T)$ be a ringed space and let $\kappa$ be an infinite cardinal such that $\kappa \geq|\mathcal{O}|$. Suppose $S$ is a sheaf on $\mathcal{O}$ and $S^{\prime} \subseteq S$ is a subpresheaf with $\left|S^{\prime}\right| \leq \kappa$. Then there exists a subpresheaf $P \subseteq S$ such that $S^{\prime} \subseteq P,|P| \leq \kappa$, and $P$ is presheaf pure in $S$. (Therefore, $P$ is stalkwise pure in $S$ as well.)

The proof in EO01] was given for a subset $Y \subseteq S(U)$ with $|Y| \leq \kappa$ (for some open subset $U \subseteq T)$, but easily generalizes to any presheaf $S^{\prime} \subseteq S$ with $\left|S^{\prime}\right| \leq \kappa$.

Next suppose we have a presheaf of $\mathcal{O}$-modules, $S$, and suppose we have a subset

$$
W \subseteq \coprod_{U \subseteq T} S(U)
$$

where $U \subseteq T$ ranges over all the open sets in $T$. We set $W_{U}=W \cap S(U)$. The set $W$ generates a presheaf $S^{\prime}$, and it is given by

$$
S^{\prime}(U)=\sum_{V \supseteq \bigcup U} \sum_{w \in W_{V}} r_{V, U}([\mathcal{O}(V)] w) .
$$

That is, $S^{\prime}(U)$ consists of all finite sums of the form

$$
r_{V_{1}, U}\left(\rho_{1} w_{1}\right)+r_{V_{2}, U}\left(\rho_{2} w_{2}\right)+\cdots+r_{V_{n}, U}\left(\rho_{n} w_{n}\right),
$$

where

$$
V_{i} \supseteq U, w_{i} \in W_{V_{i}}, \rho_{i} \in \mathcal{O}\left(V_{i}\right)
$$

and $r_{V_{i}, U}: V_{i} \rightarrow U$ are the restriction maps. It is straightforward to see that $S^{\prime}$ is a presheaf, and upon reflection it is clearly the smallest subpresheaf containing $W$. So the above is an explicit description of the intersection of all subpresheaves containing $W$. Furthermore, if $\kappa \geq|\mathcal{O}|$ is an infinite cardinal, and $|W| \leq \kappa$, then we will have $\left|S^{\prime}\right| \leq \kappa$. We use this to prove the next lemma.

Lemma 4.9. Let $\kappa$ be an infinite cardinal such that $\kappa \geq|\mathcal{O}|$ and $\kappa \geq|T|$, where $T$ is the underlying topological space. Suppose $S_{1} \stackrel{f}{\rightarrow} S_{2}$ is a surjection of $\mathcal{O}$-modules. That is, $f_{p}$ is a surjection for all $p \in T$. Let $S_{2}^{\prime} \subseteq S_{2}$ be a subpresheaf with $\left|S_{2}^{\prime}\right| \leq \kappa$. Then there exists a subpresheaf $S_{1}^{\prime} \subseteq S_{1}$ such that $f_{\mid S_{1}^{\prime}}: S_{1}^{\prime} \rightarrow S_{2}^{\prime}$ is a surjection on the stalks and $\left|S_{1}^{\prime}\right| \leq \kappa$. 
Proof. As the first step we show that for each $p \in T$ and $\beta_{p} \in\left(S_{2}^{\prime}\right)_{p}$ we can find a pair $(Z, \alpha)$, where $Z$ is an open set in $T$ containing $p$, and $\alpha \in S_{1}(Z)$ satisfies $f_{Z}(\alpha) \in S_{2}^{\prime}(Z)$ and $f_{p}\left(\alpha_{p}\right)=\beta_{p}$.

Given $p \in T$ and an element $\beta \in\left(S_{2}^{\prime}\right)_{p}$, it has the form $\beta_{p}$, where $\beta \in S_{2}^{\prime}(U)$ for some open subset $U$ of $T$ containing $p$. Noting we have $\left(S_{2}^{\prime}\right)_{p} \subseteq\left(S_{2}\right)_{p}$, we use the fact that $f_{p}$ is surjective to find $\gamma_{p} \in\left(S_{1}\right)_{p}$ for which $f_{p}\left(\gamma_{p}\right)=\beta_{p}$. Again, $\gamma \in S_{1}(V)$ for some open subset $V$ of $T$ which contains $p$. Now $f_{V}(\gamma)$ need not belong to $S_{2}^{\prime}(V)$. However, $\beta_{p}=f_{p}\left(\gamma_{p}\right)=\left[f_{V}(\gamma)\right]_{p}$, which means there exists an open set $Z \subseteq U \cap V$ such that $\beta_{\mid Z}=f_{V}(\gamma)_{\mid Z}=f_{Z}\left(\gamma_{\mid Z}\right)$. Now the pair $\left(Z, \gamma_{\mid Z}\right)$ has the desired property. Indeed $f_{Z}\left(\gamma_{\mid Z}\right)=\beta_{\mid Z} \in S_{2}^{\prime}(Z)$ and $f_{p}\left[\left(\gamma_{\mid Z}\right)_{p}\right]=\left[f_{Z}\left(\gamma_{\mid Z}\right)\right]_{p}=\left(\beta_{\mid Z}\right)_{p}=\beta_{p}$.

Using this fact we can complete the argument. For each pair $\left(p, \beta_{p}\right)$ with $p \in T$ and $\beta_{p} \in\left(S_{2}^{\prime}\right)_{p}$ choose one such pair $(Z, \alpha)$ and let $W$ be the set of all such $\alpha$ 's. Then $|W| \leq \kappa$ since $\left|\coprod_{p \in T}\left(S_{2}^{\prime}\right)_{p}\right| \leq \kappa \times \kappa=\kappa$.

Now let $S_{1}^{\prime}$ be the subpresheaf of $S_{1}$ generated by the set $W$. By construction, $W \subseteq f^{-1}\left(S_{2}^{\prime}\right)$, where $f^{-1}\left(S_{2}^{\prime}\right)$ is the preimage of $S_{2}^{\prime}$. Since $S_{1}^{\prime}$ is the smallest presheaf containing $W$, we have that $f\left(S_{1}^{\prime}\right) \subseteq S_{2}^{\prime}$. Furthermore, the restriction $f_{\mid S_{1}^{\prime}}: S_{1}^{\prime} \rightarrow S_{2}^{\prime}$ is a surjection on the stalks. Lastly, as described in the paragraph before the statement of the lemma, $\left|S_{1}^{\prime}\right| \leq \kappa$.

We prove one last lemma before we show that $(\widetilde{\mathcal{F}}, d g \widetilde{\mathcal{C}})$ is cogenerated by a set. Suppose we have a ring $R$ and a pure injection $P \subseteq F$ of $R$-modules, where $F$ is a flat module. Then $P$ and $F / P$ are both flat $R$-modules by Corollary 4.86 of [Lam99. We have an analogous result for $\mathcal{O}$-modules. To be precise, suppose $P \subseteq F$, where $F$ is a flat $\mathcal{O}$-module and $P$ is a stalkwise pure submodule. Then $P$ and $F / P$ are both flat $\mathcal{O}$-modules. Indeed, for each $p \in T$ we have a pure injection of $\mathcal{O}_{p^{-}}$-modules $P_{p} \rightarrow F_{p}$. Since $F_{p}$ is flat, $P_{p}$ and $F_{p} / P_{p} \cong(F / P)_{p}$ are flat $\mathcal{O}_{p^{-}}$ modules, too. Therefore, $P$ and $F / P$ are flat $\mathcal{O}$-modules. This sets the stage for the final lemma.

Lemma 4.10. Let $F$ be a flat chain complex of $\mathcal{O}$-modules and let $S \subseteq F$ be an exact subcomplex of $\mathcal{O}$-modules such that the subsheaves $Z_{n} S \subseteq Z_{n} F$ are stalkwise pure. Then $S$ and $F / S$ are flat complexes of $\mathcal{O}$-modules.

Proof. Since $S$ is exact, it follows from the fundamental lemma of homological algebra that $F / S$ is exact. Furthermore $Z_{n}(F / S)=Z_{n} F / Z_{n} S$ since everything is exact. So it is left to see that $Z_{n} S$ and $Z_{n} F / Z_{n} S$ are flat. But $Z_{n} S \subseteq Z_{n} F$ is stalkwise pure and $Z_{n} F$ is flat, so the result follows from the comments above.

We are now ready to show that $(\widetilde{\mathcal{F}}, d g \widetilde{\mathcal{C}})$ has enough injectives. As we already mentioned we will appeal to Lemma 4.2 and Proposition 4.1.

Proposition 4.11. Let $(\mathcal{F}, \mathcal{C})$ be the flat cotorsion pair in $\mathbf{S h}(\mathcal{O})$. Then $(\widetilde{\mathcal{F}}, d g \widetilde{\mathcal{C}})$ has enough injectives.

Proof. Let $\kappa$ be an infinite cardinal such that $\kappa \geq|\mathcal{O}|$ and $\kappa \geq|T|$. Now pick isomorphic representatives to form the set

$$
\mathcal{L}=\{F \in \tilde{\mathcal{F}}:|F| \leq \kappa\}
$$

Using this set, we will prove the hypothesis of Lemma 4.2 ,

So let $0 \neq F \in \widetilde{\mathcal{F}}$. Since $F$ is (sheaf) exact there exists some $n$ such that $0 \neq Z_{n}(F)$. Using Proposition 4.8 we can find a stalkwise pure subpresheaf $0 \neq$ $P_{n} \subseteq Z_{n} F$ with $\left|P_{n}\right| \leq \kappa$. Set $S_{n}=P_{n}$. 
Now use Lemma 4.9 to find a subpresheaf $A_{n+1}$ of $F_{n+1}$ with $\left|A_{n+1}\right| \leq \kappa$ such that $d_{\mid A_{n+1}}: A_{n+1} \rightarrow S_{n}$ is surjective on each stalk. Use Proposition 4.8 again to find a stalkwise pure subpresheaf $P_{n+1} \subseteq Z_{n+1} F$ so that

(1) $\operatorname{ker} d_{\mid A_{n+1}} \subseteq P_{n+1}$.

(2) $\left|P_{n+1}\right| \leq \kappa$.

Set $S_{n+1}=A_{n+1}+P_{n+1}$. Now $d_{\mid S_{n+1}}: S_{n+1} \rightarrow S_{n}$ is still a surjection on each stalk. Also, $\operatorname{ker}\left(d_{\mid S_{n+1}}\right)=P_{n+1}$ is stalkwise pure in $Z_{n+1} F$.

Now we sheafify the exact sequence $0 \rightarrow P_{n+1} \rightarrow S_{n+1} \rightarrow S_{n}$ of presheaves to get an exact sequence $0 \rightarrow P_{n+1}^{+} \rightarrow S_{n+1}^{+} \rightarrow S_{n}^{+} \rightarrow 0$ of $\mathcal{O}$-modules. This last sequence is exact on the right since $d_{\mid S_{n+1}}: S_{n+1} \rightarrow S_{n}$ is a surjection on each stalk. By Lemma 4.7, we have that ker $d_{\mid S_{n}^{+}}=P_{n}^{+}$is stalkwise pure in $Z_{n} F$ and ker $d_{\mid S_{n+1}^{+}}=P_{n+1}^{+}$is stalkwise pure in $Z_{n+1} F$.

Next, we use Lemma 4.9 again to find a subpresheaf $A_{n+2}$ of $F_{n+2}$ such that $\left|A_{n+2}\right| \leq \kappa$ and $d_{\mid A_{n+2}}: A_{n+2} \rightarrow P_{n+1}$ induces a surjection on the stalks. Now find a stalkwise pure subpresheaf $P_{n+2} \subseteq Z_{n+2} F$ such that

(1) $\operatorname{ker} d_{\mid A_{n+2}} \subseteq P_{n+2}$.

(2) $\left|P_{n+2}\right| \leq \kappa$.

Set $S_{n+2}=A_{n+2}+P_{n+2}$. As above $d_{\mid S_{n+2}}: S_{n+2} \rightarrow P_{n+1}$ is still a surjection on each stalk and $\operatorname{ker}\left(d_{\mid S_{n+2}}\right)=P_{n+2}$ is stalkwise pure in $Z_{n+2} F$. Again, we sheafify the exact sequence $0 \stackrel{\rightarrow}{\rightarrow} P_{n+2} \rightarrow S_{n+2} \rightarrow P_{n+1}$ of presheaves to get an exact sequence of $0 \rightarrow P_{n+2}^{+} \rightarrow S_{n+2}^{+} \rightarrow P_{n+1}^{+} \rightarrow 0$ of $\mathcal{O}$-modules for which ker $d_{\mid S_{n+2}^{+}}=$ $P_{n+2}^{+}$is stalkwise pure in $Z_{n+2} F$. "Pasting" this sequence together with the above sequence at $P_{n+1}^{+}$, we have the sheaf exact sequence

$$
0 \rightarrow P_{n+2}^{+} \rightarrow S_{n+2}^{+} \rightarrow S_{n+1}^{+} \rightarrow S_{n}^{+} \rightarrow 0 \text {. }
$$

In this way we continue by induction to build a (sheaf) exact subcomplex of $\mathcal{O}$-modules

$$
S^{+}=\cdots \rightarrow S_{n+2}^{+} \rightarrow S_{n+1}^{+} \rightarrow S_{n}^{+} \rightarrow 0 \rightarrow 0 \rightarrow \cdots
$$

with each cycle module $Z_{i}\left(S^{+}\right) \subseteq Z_{i}(F)$ a stalkwise pure subsheaf. By Lemma 4.10 we see that $S^{+}$and $F / S^{+}$are both flat complexes. Since $\left|S^{+}\right| \leq \kappa$ we have $S^{+} \in \mathcal{L}$, and we are done.

Combining Proposition 4.11 with Corollary 3.8 we get the following corollary.

Corollary 4.12. Let $(\mathcal{F}, \mathcal{C})$ be the flat cotorsion pair in $\mathbf{S h}(\mathcal{O})$. Then we have an induced model category structure on $\mathbf{C h}(\mathcal{O})$, where the weak equivalences are the homology isomorphisms. The cofibrations are monomorphisms whose cokernels are in $d g \widetilde{\mathcal{F}}$ and the fibrations are the epimorphisms whose kernels are in $d g \widetilde{\mathcal{C}}$.

\section{The FLAT MODEL STRUCTURE IS MONOIDAL}

Again we let $\mathbf{C h}(\mathcal{O})$ be the category of chain complexes of sheaves of $\mathcal{O}$-modules, where $(\mathcal{O}, T)$ is any fixed ringed space. In this section we show that the flat model structure obtained in section 4 is actually monoidal. It follows that the sheaf tensor product on $\mathbf{C h}(\mathcal{O})$ descends to a tensor product on the homotopy category. Monoidal model categories are defined and studied in chapter four of [Hov99. Whenever we are working in a symmetric monoidal category and we have compatible classes $\mathcal{Q}$ and $\mathcal{R}$ which induce a model structure as in the statement of Theorem [2.1, we can use Theorem 7.2 of [Hov02] to see if the resulting model 
structure is monoidal. This theorem is simply a list of criteria which the classes $\mathcal{Q}, \mathcal{R}, \mathcal{Q} \cap \mathcal{E}$, and $\mathcal{R} \cap \mathcal{E}$ must satisfy. For our situation these are the classes of dg-flat, dg-cotorsion, flat and cotorsion complexes, respectively. The following proposition is simply the statement of Hovey's criteria with the language specialized to the flat model structure of Corollary 4.12

Proposition 5.1 (Hovey). Let $(\mathcal{F}, \mathcal{C})$ be the flat cotorsion theory on $\boldsymbol{S h}(\mathcal{O})$. The induced model structure on $\boldsymbol{C h}(\mathcal{O})$ is monoidal (with respect to the usual tensor product of complexes) if the following criteria are met:

(1) Every cofibration is a pure injection in each degree.

(2) If $X$ and $Y$ are dg-flat, then $X \otimes Y$ is dg-flat.

(3) If $X$ is dg-flat and $Y$ is flat, then $X \otimes Y$ is flat.

(4) The unit of the monoidal structure is dg-flat.

The monoidal structure on $\mathbf{S h}(\mathcal{O})$ is given by the sheaf tensor product which we defined in section 2, The closed structure is given by the "sheaf of restrictions" which we will now describe. For any open $U \subseteq T$, we may restrict $\mathcal{O}$ to get a sheaf of rings, denoted $\left.\mathcal{O}\right|_{U}$, on the space $U$. Similarly, given an $\mathcal{O}$-module $S$, we may restrict to get $\left.S\right|_{U}$, an $\left.\mathcal{O}\right|_{U}$-module. For any two $\mathcal{O}$-modules $S_{1}$ and $S_{2}$, the collection of $\left.\mathcal{O}\right|_{U}$-homomorphisms $\left.\left.S_{1}\right|_{U} \rightarrow S_{2}\right|_{U}$, denoted $\mathbf{S h}\left(\left.\mathcal{O}\right|_{U}\right)\left(\left.S_{1}\right|_{U},\left.S_{2}\right|_{U}\right)$, is an abelian group. In fact, it is actually an $\mathcal{O}(U)$-module. Indeed, given $r \in \mathcal{O}(U)$ and $f=\left\{f_{V}\right\}_{V \subseteq U}:\left.\left.S_{1}\right|_{U} \rightarrow S_{2}\right|_{U}$ we define $r f=\left\{(r f)_{V}\right\}_{V \subseteq U}$ by $(r f)_{V}(x)=$ $\left.r\right|_{V}\left(f_{V}(x)\right)$ for $x \in S_{1}(V)$. One can check that $r f$ is a map of $\left.\mathcal{O}\right|_{U}$-modules and that $\mathbf{S h}\left(\left.\mathcal{O}\right|_{U}\right)\left(\left.S_{1}\right|_{U},\left.S_{2}\right|_{U}\right)$ is an $\mathcal{O}(U)$-module. The sheaf of restrictions is the $\mathcal{O}$ module, denoted $\mathcal{H o m}\left(S_{1}, S_{2}\right)$, defined by

$$
\mathcal{H o m}\left(S_{1}, S_{2}\right)(U)=\mathbf{S h}(U)\left(\left.S_{1}\right|_{U},\left.S_{2}\right|_{U}\right) .
$$

The adjointness property states that for $\mathcal{O}$-modules $S_{1}, S_{2}$ and $S_{3}$ we have

$$
\mathcal{H o m}\left(S_{1} \otimes_{\mathcal{O}} S_{2}, S_{3}\right) \cong \mathcal{H o m}\left(S_{1}, \mathcal{H o m}\left(S_{2}, S_{3}\right)\right) \text {. }
$$

These functors extend to make $\mathbf{C h}(\mathcal{O})$ a closed symmetric monoidal category as well. Here the monoidal structure is given by the chain complex tensor product $X \otimes Y$, which in degree $n$ is given by $(X \otimes Y)_{n}=\bigoplus_{i+j=n} X_{i} \otimes_{\mathcal{O}} Y_{j}$. The differential is defined on the $(i, j)$-component by $d_{X_{i}} \otimes_{\mathcal{O}} 1_{Y_{j}}+(-1)^{i} 1_{X_{i}} \otimes_{\mathcal{O}} d_{Y_{j}}$. The closed structure will be denoted by $\mathcal{H o m}(X, Y)$ and is given in degree $n$ by

$$
[\mathcal{H o m}(X, Y)]_{n}=\prod_{k \in \mathbb{Z}} \mathcal{H o m}\left(X_{k}, Y_{k+n}\right) .
$$

The differential is a map of sheaves

$$
\prod_{k \in \mathbb{Z}} \mathcal{H o m}\left(X_{k}, Y_{k+n}\right) \rightarrow \prod_{k \in \mathbb{Z}} \mathcal{H o m}\left(X_{k}, Y_{k+n-1}\right) .
$$

Given the open set $U \subseteq T$, it acts on the $\mathcal{O}$-modules

$$
\prod_{k \in \mathbb{Z}} \mathbf{S h}(U)\left(\left.X_{k}\right|_{U},\left.Y_{k+n}\right|_{U}\right) \rightarrow \prod_{k \in \mathbb{Z}} \mathbf{S h}(U)\left(\left.X_{k}\right|_{U},\left.Y_{k+n-1}\right|_{U}\right)
$$

by

$$
\left\{f_{k}\right\} \mapsto d_{k+n} f_{k}-(-1)^{n} f_{k-1} d_{k} .
$$

In particular, for any open $U \subseteq T, \mathcal{H o m}(X, Y)(U)$ is a chain complex in the category $\mathbf{C h}(\mathcal{O}(U))$. This complex has the property that it is exact iff every chain 
map $f:\left.X\right|_{U} \rightarrow \Sigma^{n}\left(\left.Y\right|_{U}\right)$ is homotopic to zero (where $\Sigma^{n}$ is the suspension functor which "shifts the complex up" $n$-degrees and makes the differentials negative when $n$ is odd). In particular, if $\mathcal{H o m}(X, Y)$ is presheaf exact, then the "global sections complex" $\operatorname{Hom}(X, Y)(T)$ is exact and so every chain map $f: X \rightarrow \Sigma^{n} Y$ is homotopic to zero. We will denote this global sections complex by $\operatorname{Hom}(X, Y)$. The adjointness property states that for complexes of sheaves $X, Y$ and $Z$ we have

$$
\mathcal{H o m}(X \otimes Y, Z) \cong \mathcal{H o m}(X, \mathcal{H o m}(Y, Z)) .
$$

Recall that the abelian group $\mathbb{Q} / \mathbb{Z}$ is an injective cogenerator. As a result it has the following property: Given any sequence of abelian groups (or $R$-modules)

$$
0 \rightarrow L \rightarrow M \rightarrow N \rightarrow 0
$$

if

$$
0 \rightarrow \operatorname{Hom}(N, \mathbb{Q} / \mathbb{Z}) \rightarrow \operatorname{Hom}(M, \mathbb{Q} / \mathbb{Z}) \rightarrow \operatorname{Hom}(L, \mathbb{Q} / \mathbb{Z}) \rightarrow 0
$$

is exact, then

$$
0 \rightarrow L \rightarrow M \rightarrow N \rightarrow 0
$$

must be exact too. We would like a sheaf with the same property. To construct one, start by forming for each $p \in T$, the "skyscraper" sheaf $\mathcal{S}_{p, \mathbb{Q} / \mathbb{Z}}$. It is defined by assigning an open set $U \subseteq T$ to $\mathbb{Q} / \mathbb{Z}$ if $p \in U$ and 0 otherwise. The skyscrapers $\mathcal{S}_{p, \mathbb{Q} / \mathbb{Z}}$ have the property that for any sheaf $F$ we have natural isomorphisms

$$
\mathbf{S h}\left(F, \mathcal{S}_{p, \mathbb{Q} / \mathbb{Z}}\right) \cong \mathbf{A b}\left(F_{p}, \mathbb{Q} / \mathbb{Z}\right) \text {. }
$$

From this it follows that each $\mathcal{S}_{p, \mathbb{Q} / \mathbb{Z}}$ is injective and so the product

$$
\mathcal{Q}=\prod_{p \in T} \mathcal{S}_{p, \mathbb{Q} / \mathbb{Z}}
$$

is injective too. We claim that $\mathcal{Q}$ is an injective cogenerator. By Proposition IV.6.5 of [Sten75] it is enough to show that given any sheaf $F \neq 0$, there exists a nonzero map $F \rightarrow \mathcal{Q}$. But $F \neq 0$ implies there exists $p \in T$ such that $F_{p} \neq 0$. Therefore there exists a nonzero map $F_{p} \rightarrow \mathbb{Q} / \mathbb{Z}$, and this gives rise to a nonzero map $F \rightarrow$ $\mathcal{S}_{p, \mathbb{Q} / \mathbb{Z}}$. This in turn induces a nonzero map $F \rightarrow \mathcal{Q}$.

In the same way that $\mathbf{A b}(M, \mathbb{Q} / \mathbb{Z})$ is an $R$-module whenever $M$ is an $R$-module, $\mathcal{H o m}(S, \mathcal{Q})$ is an $\mathcal{O}$-module whenever $S$ is an $\mathcal{O}$-module. Also, the sheaf $\mathcal{H o m}(S, \mathcal{Q})$ is flasque. Recall that a sheaf, or $\mathcal{O}$-module, $F$, is called flasque if whenever we have open sets $U \subseteq V$, the restriction map $F(V) \rightarrow F(U)$ is a surjection. It is not hard to show that for any sheaf $S, \mathcal{H o m}(S, I)$ is flasque whenever $I$ is injective. So in particular, the sheaf $\mathcal{H o m}(S, \mathcal{Q})$ is flasque.

Lemma 5.2. Let $0 \rightarrow F \rightarrow G \rightarrow H \rightarrow 0$ be a sequence of sheaves (or $\mathcal{O}$-modules) on $T$. If

$$
0 \rightarrow \mathcal{H o m}(H, \mathcal{Q}) \rightarrow \mathcal{H o m}(G, \mathcal{Q}) \rightarrow \mathcal{H o m}(F, \mathcal{Q}) \rightarrow 0
$$

is a sheaf exact sequence, then $0 \rightarrow F \rightarrow G \rightarrow H \rightarrow 0$ must also be a sheaf exact sequence.

Proof. Of course we only need to prove the lemma for sequences of sheaves, since having an $\mathcal{O}$-module structure on a sheaf does not change the notion of exactness. So suppose $0 \rightarrow \mathcal{H o m}(H, \mathcal{Q}) \rightarrow \mathcal{H o m}(G, \mathcal{Q}) \rightarrow \mathcal{H o m}(F, \mathcal{Q}) \rightarrow 0$ is sheaf exact. Since $\operatorname{Hom}(H, \mathcal{Q})$ is flasque the sequence must actually be presheaf exact Har77. In particular,

$$
0 \rightarrow \mathbf{S h}(H, \mathcal{Q}) \rightarrow \mathbf{S h}(G, \mathcal{Q}) \rightarrow \mathbf{S h}(F, \mathcal{Q}) \rightarrow 0
$$




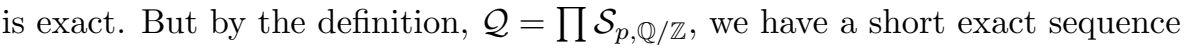

$$
0 \rightarrow \prod_{p \in T} \mathbf{S h}\left(H, \mathcal{S}_{p, \mathbb{Q} / \mathbb{Z}}\right) \rightarrow \prod_{p \in T} \mathbf{S h}\left(G, \mathcal{S}_{p, \mathbb{Q} / \mathbb{Z}}\right) \rightarrow \prod_{p \in T} \mathbf{S h}\left(F, \mathcal{S}_{p, \mathbb{Q} / \mathbb{Z}}\right) \rightarrow 0 .
$$

Therefore, for each $p$ we have the short exact sequence

$$
0 \rightarrow \mathbf{S h}\left(H, \mathcal{S}_{p, \mathbb{Q} / \mathbb{Z}}\right) \rightarrow \mathbf{S h}\left(G, \mathcal{S}_{p, \mathbb{Q} / \mathbb{Z}}\right) \rightarrow \mathbf{S h}\left(F, \mathcal{S}_{p, \mathbb{Q} / \mathbb{Z}}\right) \rightarrow 0 .
$$

Using the natural isomorphism

$$
\operatorname{Sh}\left(F, \mathcal{S}_{p, \mathbb{Q} / \mathbb{Z}}\right) \cong \mathbf{A b}\left(F_{p}, \mathbb{Q} / \mathbb{Z}\right),
$$

we see that each sequence

$$
0 \rightarrow \mathbf{A b}\left(H_{p}, \mathbb{Q} / \mathbb{Z}\right) \rightarrow \mathbf{A b}\left(G_{p}, \mathbb{Q} / \mathbb{Z}\right) \rightarrow \mathbf{A b}\left(F_{p}, \mathbb{Q} / \mathbb{Z}\right) \rightarrow 0
$$

is exact. Since $\mathbb{Q} / \mathbb{Z}$ is a cogenerator, we have a short exact sequence

$$
0 \rightarrow F_{p} \rightarrow G_{p} \rightarrow H_{p} \rightarrow 0
$$

for each $p$. This means $0 \rightarrow F \rightarrow G \rightarrow H \rightarrow 0$ is sheaf exact.

We should point out that if the functor $\mathcal{H o m}(-, J)$ is sheaf exact, it does not necessarily imply that the $\mathcal{O}$-module $J$ is injective. Of course, by definition an $\mathcal{O}$ module $I$ is injective if $\operatorname{Sh}(\mathcal{O})(-, I)$ is exact. It turns out that $I$ is injective if and only if $\mathcal{H o m}(-, I)$ is presheaf exact. Given $J$, it is possible for $\mathcal{H o m}(-, J)$ to be sheaf exact and yet $J$ neither injective nor even "locally injective" in the sense that for each $p \in T$ there exists an open set $U$ containing $p$ for which $\left.J\right|_{U}$ is injective. However, if we also know that $\operatorname{Hom}(F, J)$ is flasque for any $\mathcal{O}$-module $F$, then we know that indeed $J$ must be injective. For if

$$
0 \rightarrow \mathcal{H o m}(H, J) \rightarrow \mathcal{H o m}(G, J) \rightarrow \mathcal{H o m}(F, J) \rightarrow 0
$$

is sheaf exact and $\mathcal{H o m}(H, J)$ is flasque, then it follows that the sequence is presheaf exact [Har77, Section 2.1]. We use this fact in the proof of the next lemma which generalizes a classical result about $R$-modules which is due to Lambek. (See Theorem 4.9 of [Lam99.)

Lemma 5.3. An $\mathcal{O}$-module $F$ is flat if and only if the $\mathcal{O}$-module $\operatorname{Hom}(F, \mathcal{Q})$ is injective.

Proof. Suppose $F$ is flat. Then $-\otimes_{\mathcal{O}} F$ is sheaf exact and so is $\mathcal{H}$ om $(-, \mathcal{Q})$. Composing functors gives us a sheaf exact functor $\mathcal{H o m}\left(-\otimes_{\mathcal{O}} F, \mathcal{Q}\right)$. Since $\mathcal{H o m}\left(S \otimes_{\mathcal{O}} F, \mathcal{Q}\right)$ is flasque for any $S, \mathcal{H o m}\left(-\otimes_{\mathcal{O}} F, \mathcal{Q}\right)$ must be presheaf exact. Using adjointness, the functor $\mathcal{H o m}(-, \mathcal{H o m}(F, \mathcal{Q}))$ is presheaf exact. Therefore, $\mathcal{H o m}(F, \mathcal{Q})$ is injective.

Conversely, let $\mathcal{H o m}(F, \mathcal{Q})$ be injective. Then

$$
\mathcal{H o m}(-, \mathcal{H o m}(F, \mathcal{Q})) \cong \mathcal{H o m}\left(-\otimes_{\mathcal{O}} F, \mathcal{Q}\right)
$$

is exact. (In fact, it is presheaf exact.) So given any sheaf exact sequence

$$
0 \rightarrow G^{\prime} \rightarrow G \rightarrow G^{\prime \prime} \rightarrow 0
$$

we get another sheaf exact sequence

$$
0 \rightarrow \mathcal{H o m}\left(G^{\prime \prime} \otimes_{\mathcal{O}} F, \mathcal{Q}\right) \rightarrow \mathcal{H o m}\left(G \otimes_{\mathcal{O}} F, \mathcal{Q}\right) \rightarrow \mathcal{H o m}\left(G^{\prime} \otimes_{\mathcal{O}} F, \mathcal{Q}\right) \rightarrow 0 .
$$

Using Lemma 5.2 we see that $0 \rightarrow G^{\prime} \otimes_{\mathcal{O}} F \rightarrow G \otimes_{\mathcal{O}} F \rightarrow G^{\prime \prime} \otimes_{\mathcal{O}} F \rightarrow 0$ is sheaf exact, and so $F$ must be flat. 
With the sheaf $\mathcal{Q}$ on hand we go back to working with complexes of $\mathcal{O}$-modules. If $X$ is a complex of $\mathcal{O}$-modules, then applying the functor $\mathcal{H o m}(-, \mathcal{Q})$ gives us another chain complex of $\mathcal{O}$-modules which we denote $X^{*}$. We point out that if $S(\mathcal{Q})$ is the chain complex consisting only of $\mathcal{Q}$ in degree zero, then $X^{*}=\mathcal{H}$ om $(X, S(\mathcal{Q}))$. A useful fact is that if $E$ is a sheaf exact complex, then $E^{*}$ must be presheaf exact. For $E^{*}$ is certainly sheaf exact, and yet all entries and cycles are flasque.

If we take $\mathcal{A}$ to be the class of all $\mathcal{O}$-modules and $\mathcal{I}$ to be the class of all injective $\mathcal{O}$-modules, then $(\mathcal{A}, \mathcal{I})$ is a complete cotorsion pair since $\operatorname{Sh}(\mathcal{O})$ has enough injectives. Applying Definition 2.2 gives us the notion of an injective complex and a $d g$-injective complex. An injective complex is a complex $I$ which is sheaf exact and has each cycle $Z_{n} I$ injective. $I$ is a dg-injective complex if each $I_{n}$ is an injective $\mathcal{O}$-module and every chain map $X \rightarrow I$ is homotopic to zero (or equivalently, the global sections complex $\operatorname{Hom}(X, I)$ is exact for any $X)$. The class $\widetilde{\mathcal{A}}$ turns out to be $\mathcal{E}$, the class of exact complexes, and $d g \widetilde{\mathcal{A}}$ turns out to be the class of all complexes since any map into an injective $\mathcal{O}$-module is always homotopic to zero. We use this notation in the proof of the next lemma. An analogous result about chain complexes of $R$-modules is due to Enochs and Garcia-Rozas and can be found as Theorem 4.1.3 of GR99.

Lemma 5.4. Let $F$ and $I$ be chain complexes.

(1) $F$ is flat if and only if $F^{*}$ is injective.

(2) $I$ is injective if and only if $I$ is both exact and dg-injective.

Proof. The first statement follows from Lemmas 5.2 and 5.3 .

For the second statement we use the fact that the category of chain complexes of $\mathcal{O}$-modules has enough injectives. (See for example, Proposition 3.2 of Gil04.) In the language of Definition 2.2 this just means that the cotorsion pair $(d g \widetilde{\mathcal{A}}, \widetilde{\mathcal{I}})$ induced by $(\mathcal{A}, \mathcal{I})$ is complete. So Lemma 3.14 of Gil04 tells us that $d g \widetilde{\mathcal{I}} \cap \mathcal{E}=\widetilde{\mathcal{I}}$ where $\mathcal{E}$ is the class of exact complexes.

Lemma 5.4 reveals a useful duality between flat complexes and injective complexes. A similar relationship exists between exact complexes and cotorsion complexes. This will be given next as Lemma 5.5. Both facts are needed to prove Proposition 5.6 which is the key to showing the flat model structure is monoidal.

Lemma 5.5. Let $E$ be a chain complex of $\mathcal{O}$-modules. $E$ is exact if and only if $E^{*}$ is cotorsion.

Proof. By Lemma 5.2, $E$ is exact if and only if $E^{*}$ is exact. Since a complex is cotorsion if it is exact and all its cycles are cotorsion, the result will follow by showing $\operatorname{Hom}(S, \mathcal{Q})$ is cotorsion for any $\mathcal{O}$-module $S$.

So let $S$ be any $\mathcal{O}$-module and let $F$ be an arbitrary flat $\mathcal{O}$-module. We wish to show $\operatorname{Ext}_{\mathcal{O}}^{1}(F, \mathcal{H o m}(S, \mathcal{Q}))=0$. We start by letting

$$
\cdots \rightarrow F_{2} \rightarrow F_{1} \rightarrow F_{0} \stackrel{\epsilon}{\rightarrow} S \rightarrow 0=\mathcal{F}_{\bullet} \stackrel{\epsilon}{\rightarrow} S
$$

be a resolution of $S$ with each $F_{n}$ flat. By applying the functor $\mathcal{H o m}(-, \mathcal{Q})$ we get a complex

$$
0 \rightarrow \mathcal{H o m}(S, \mathcal{Q}) \stackrel{\epsilon^{*}}{\longrightarrow} \mathcal{H o m}\left(\mathcal{F}_{\bullet}, \mathcal{Q}\right)
$$


which is an injective coresolution of $\mathcal{H o m}(S, \mathcal{Q})$ by Lemma 5.3. So we can compute $\operatorname{Ext}_{\mathcal{O}}^{1}(F, \mathcal{H o m}(S, \mathcal{Q}))$ by applying the functor $\operatorname{Sh}(\mathcal{O})(F,-)$ to the complex

$$
0 \rightarrow \mathcal{H o m}\left(\mathcal{F}_{\bullet}, \mathcal{Q}\right)
$$

and taking the first cohomology $\mathcal{O}$-module. I.e.,

$$
\operatorname{Ext}_{\mathcal{O}}^{1}(F, \mathcal{H o m}(S, \mathcal{Q}))=H^{1}\left(\operatorname{Sh}(\mathcal{O})\left(F, \mathcal{H o m}\left(\mathcal{F}_{\bullet}, \mathcal{Q}\right)\right)\right) .
$$

But using adjoint associativity

$$
\operatorname{Sh}(\mathcal{O})\left(F, \mathcal{H o m}\left(\mathcal{F}_{\bullet}, \mathcal{Q}\right)\right) \cong \mathbf{S h}\left(F \otimes_{\mathcal{O}} \mathcal{F}_{\bullet}, \mathcal{Q}\right)
$$

and since $F \otimes_{\mathcal{O}}-$ and $\mathbf{S h}(-, \mathcal{Q})$ are both exact, we see that $H^{n}\left(\mathbf{S h}\left(F \otimes_{\mathcal{O}} \mathcal{F}_{\bullet}, \mathcal{Q}\right)\right)=0$ for $n>0$. So $\operatorname{Ext}_{\mathcal{O}}^{n}(F, \mathcal{H o m}(S, \mathcal{Q}))=0$ for $n>0$.

Proposition 5.6. A complex of $\mathcal{O}$-modules $F$ is dg-flat if and only if $F_{n}$ is flat for each $n$, and $F \otimes_{\mathcal{O}} E$ is exact for each exact complex $E$.

Proof. $(\Rightarrow)$ Let $F$ be dg-flat and $E$ any exact complex. We wish to show $F \otimes E$ is exact. By Lemma 5.5, $E^{*}$ is cotorsion. So the global sections complex

$$
\operatorname{Hom}\left(F, E^{*}\right)=\mathcal{H o m}\left(F, E^{*}\right)(T)
$$

is exact. But using adjoint associativity

$$
\mathcal{H o m}\left(F, E^{*}\right)=\mathcal{H o m}(F, \mathcal{H o m}(E, S(\mathcal{Q}))) \cong \mathcal{H o m}(F \otimes E, S(\mathcal{Q}))=(F \otimes E)^{*} .
$$

So the global sections complex

$$
(F \otimes E)^{*}(T)=\cdots \rightarrow \mathbf{S h}\left((F \otimes E)_{n}, \mathcal{Q}\right) \rightarrow \mathbf{S h}\left((F \otimes E)_{n+1}, \mathcal{Q}\right) \rightarrow \cdots
$$

must be an exact sequence (of $\mathcal{O}(T)$-modules). Now one argues as in the proof of Lemma 5.2 to conclude that $F \otimes E$ is exact.

$(\Leftarrow)$ Suppose that we have a chain complex $F$ in which every $F_{n}$ is a flat $\mathcal{O}$ module and $F \otimes E$ is exact for each exact complex $E$. By Corollary 3.7 we can find a short exact sequence

$$
0 \rightarrow F \rightarrow C \rightarrow F^{\prime} \rightarrow 0,
$$

where $C$ is cotorsion and $F^{\prime}$ is dg-flat.

We claim that $C$ is in fact flat. Using Lemma 5.4, it is enough to show that $C^{*}$ is exact and dg-injective. Certainly $C^{*}$ is exact since $C$ is exact. Also $C_{n}$ is flat since it is an extension of $F_{n}$ and $F_{n}^{\prime}$. This tells us that $\left(C^{*}\right)_{n}$ is injective. To finish showing $C^{*}$ is dg-injective we need to show that every map $E \rightarrow C^{*}$, where $E$ is exact, is homotopic to zero. This follows from showing that the global sections complex $\operatorname{Hom}\left(E, C^{*}\right)=\operatorname{Hom}\left(E, C^{*}\right)(T)$ is exact.

Now for any complex $E$ we have a short exact sequence

$$
0 \rightarrow F \otimes E \rightarrow C \otimes E \rightarrow F^{\prime} \otimes E \rightarrow 0
$$

since $0 \rightarrow F \rightarrow C \rightarrow F^{\prime} \rightarrow 0$ is "degreewise pure". Furthermore, if $E$ is exact, then by hypothesis $F \otimes E$ is exact, and since we have proved the $(\Rightarrow)$ direction of the Proposition, $F^{\prime} \otimes E$ is exact too. Therefore, by the fundamental lemma of homological algebra, $C$ also has the property that $C \otimes E$ is exact whenever $E$ is exact.

Using this property, we go back to the problem of showing that the global sections complex $\operatorname{Hom}\left(E, C^{*}\right)$ is exact whenever $E$ is exact: For such an $E$, we have

$$
\mathcal{H o m}\left(E, C^{*}\right)=\mathcal{H o m}(E, \mathcal{H o m}(C, S(\mathcal{Q}))) \cong \mathcal{H o m}(E \otimes C, S(\mathcal{Q}))=(E \otimes C)^{*},
$$


and the complex on the right is clearly exact. In fact, $(E \otimes C)^{*}$ must be presheaf exact by the comments made after the proof of Lemma 5.3. Therefore, the global sections complex $\operatorname{Hom}\left(E, C^{*}\right)=\mathcal{H o m}\left(E, C^{*}\right)(T)$ is exact. This concludes the proof that $C$ is flat.

Now for any cotorsion complex $C^{\prime}$, the sequence

$$
0 \rightarrow \operatorname{Hom}\left(F^{\prime}, C^{\prime}\right) \rightarrow \operatorname{Hom}\left(C, C^{\prime}\right) \rightarrow \operatorname{Hom}\left(F, C^{\prime}\right) \rightarrow 0
$$

is exact. Indeed, in degree $n$ we have the exact sequence

$$
\prod_{k \in \mathbb{Z}} \operatorname{Sh}(\mathcal{O})\left(C_{k}, C_{k+n}^{\prime}\right) \rightarrow \prod_{k \in \mathbb{Z}} \operatorname{Sh}(\mathcal{O})\left(F_{k}, C_{k+n}^{\prime}\right) \rightarrow \prod_{k \in \mathbb{Z}} \operatorname{Ext}_{\mathcal{O}}^{1}\left(F_{k}^{\prime}, C_{k+n}^{\prime}\right)=0
$$

Since $F^{\prime}$ and $C$ are both dg-flat we see that $\operatorname{Hom}\left(F^{\prime}, C^{\prime}\right)$ and $\operatorname{Hom}\left(C, C^{\prime}\right)$ are exact. Thus the fundamental lemma of homological algebra implies $\operatorname{Hom}\left(F, C^{\prime}\right)$ is exact, so that $F$ is dg-flat.

Finally, we may now prove that the flat model structure is monoidal.

Theorem 5.7. Let $(\mathcal{F}, \mathcal{C})$ be the flat cotorsion pair in the category $\boldsymbol{S h}(\mathcal{O})$. Then the induced model structure on $\boldsymbol{C h}(\mathcal{O})$ is monoidal with respect to the usual tensor product of chain complexes.

Proof. We use Hovey's criteria listed in Proposition 5.1.

1) Cofibrations are monomorphisms with dg-flat cokernels. In particular, the cokernel is flat in each degree. Therefore each cofibration is a pure injection in each degree. (See for example, the proof of Lemma XVI.3.1 in Lan97.)

2) Let $X, Y$ be dg-flat. We wish to show $X \otimes Y$ is dg-flat. It is easy to see that $(X \otimes Y)_{n}$ is flat for each $n$. Now given an exact complex $E$, we know by Proposition 5.6 that $Y \otimes E$ is exact, and likewise $X \otimes(Y \otimes E)$ is exact. But then $(X \otimes Y) \otimes E$ is exact and using Proposition 5.6 again we get that $X \otimes Y$ is dg-flat.

3) Let $X$ be dg-flat and $Y$ flat. We wish to show $X \otimes Y$ is flat. Recall that a complex is flat if and only if it is dg-flat and exact. So by 2) above, $X \otimes Y$ is dg-flat. But since $Y$ is exact, $X \otimes Y$ is also exact. Therefore $X \otimes Y$ is flat.

4) The unit of the monoidal structure is $S(\mathcal{O})$, the complex consisting of $\mathcal{O}$ in degree zero and 0 otherwise. Since $\mathcal{O}$ is a flat $\mathcal{O}$-module, $S(\mathcal{O})$ is dg-flat by Lemma 3.4 of Gil04.

\section{REFERENCES}

[Ald01] S. Tempest Aldrich, Edgar E. Enochs, Luis Oyonarte, and J.R. García Rozas, Covers and envelopes in Grothendieck categories: flat covers of complexes with applications, Journal of Algebra 243, 2001, pp. 615-630. MR.1850650 (2002i:18010)

[BBE01] L. Bican, R. El Bashir and E. Enochs, All modules have flat covers, Bull. London Math Soc., vol. 33, no. 4, 2001, pp. 385-390. MR1832549 (2002e:16002)

[DS95] W.G. Dwyer and J. Spalinski, Homotopy theories and model categories, Handbook of algebraic topology (Amsterdam), North-Holland, Amsterdam, 1995, pp. 73-126. MR.1361887 (96h:55014)

[ET01] Paul C. Eklof and Jan Trlifaj, How to make Ext vanish, Bull. London Math Soc., vol. 33, no. 1, 2001, pp. 41-51. MR1798574 (2001i:16015)

[EEGO] E. Enochs, S. Estrada, J.R. García Rozas and L. Oyonarte, Flat covers in the category of quasi-coherent sheaves over the projective line, Communications in Algebra, vol. 32, no. 4, 2004, pp. 1497-1508. MR.2100370 (2005m:14024)

[EJ01] E. Enochs and O. Jenda, Relative homological algebra, De Gruyter Expositions in Mathematics no. 30, Walter De Gruyter, New York, 2001. MR1753146 (2001h:16013) 
[EO01] E. Enochs and L Oyonarte, Flat covers and cotorsion envelopes of sheaves, Proceedings of the American Mathematical Society vol. 130, no. 5, 2002, pp. 1285-1292. MR 1879949 (2003d:18023)

[EGR97] E. Enochs and J.R. García Rozas, Tensor products of chain complexes, Math J. Okayama Univ. vol. 39, 1997, pp. 19-42. MR.1680739 (2001b:16006)

[GR99] J. R. García Rozas, Covers and envelopes in the category of complexes of modules, Research Notes in Mathematics no. 407, Chapman \& Hall/CRC, Boca Raton, FL, 1999. MR.1693036 (2001i:16009)

[Gil04] James Gillespie, The flat model structure on $C h(R)$, Transactions of the American Mathematical Society, vol. 356, no. 8, 2004, pp. 3369-3390. MR.2052954 (2005b:18020)

[Gri99] Pierre Antoine Grillet, Algebra, Pure and Applied Mathematics, John Wiley \& Sons, New York, 1999. MR1689024 (2000g:20001)

[Gro57] A. Grothendieck, Sur quelques points d'algèbre homologique, Tôhoku Math J. (2), 9, 1957, pp. 119-221. MR0102537 (21:1328)

[Har77] Robin Hartshorne, Algebraic Geometry, Graduate Texts in Mathematics, vol. 52, Springer-Verlag, New York, 1977. MR0463157 (57:3116)

[Har66] R. Hartshorne, Residues and Duality, Lecture Notes in Mathematics, Springer-Verlag, 1966. MR0222093 (36:5145)

[Hov01] Mark Hovey, Model category structures on chain complexes of sheaves, Transactions of the American Mathematical Society, vol. 353, no. 6, 2001, 2441-2457. MR1814077 (2002a:18015)

[Hov02] Mark Hovey, Cotorsion theories, model category structures, and representation theory, Mathematische Zeitschrift, vol. 241, 2002, 553-592. MR1938704 (2003m:55027)

[Hov99] Mark Hovey, Model categories, Mathematical Surveys and Monographs, vol. 63, American Mathematical Society, 1999. MR:1650134 (99h:55031)

[Joy84] A. Joyal, Letter to A. Grothendieck, 1984.

[Lam99] T.Y. Lam, Lectures on Modules and Rings, Graduate Texts in Mathematics, vol. 189, Springer-Verlag, New York, 1999. MR.1653294 (99i:16001)

[Lit82] Shigero Litaka, Algebraic Geometry, Graduate Texts in Mathemamtics, vol. 76, SpringerVerlag, New York, 1982. MR0637060 (84j:14001)

[Lan97] S. Lang, Algebra, Addison-Wesley, third edition, 1997. MR0197234 (33:5416)

[Mac71] Saunders MacLane, Categories for the working mathematician, Graduate Texts in Mathematatics, vol. 5, Springer-Verlag, New York, second edition, 1998. MR1712872 (2001j:18001)

[Qui67] Daniel G. Quillen, Homotopical algebra, Lecture Notes in Mathematics, no. 43, SpringerVerlag, 1967. MR0223432 (36:6480)

[Span66] Edwin H. Spanier, Algebraic Topology, McGraw-Hill series in higher mathematics, McGraw-Hill, New York, 1966. MR0210112 (35:1007)

[Sten75] Bo Stenström, Rings of Quotients, Die Grundlehren der mathematischen Wissenschaften in Einzeldarstellungen Band 217, Springer-Verlag, New York, 1975. MR0389953 $(52: 10782)$

[Wis91] Robert Wisbauer, Foundations of module and ring theory, Algebra, Logic and Applications series, vol. 3, Gordon and Breach Science Publishers, 1991. MR1144522 (92i:16001)

[Xu96] Jinzhong Xu, Flat covers of modules, Lecture Notes in Mathematics, no. 1634, SpringerVerlag, Berlin, 1996. MR1438789 (98b:16003)

Department of Mathematics, 4000 University Drive, Penn State McKeesport, McKeesport, Pennsylvania 15132-7698

E-mail address: jrg21@psu.edu 